\title{
Some proprioceptive influences on the spatial displacement component of the oculogyral illusion
}

\author{
JOHN N. EVANOFF and JAMES R. LACKNER \\ Brandeis University, Waltham, Massachusetts
}

\begin{abstract}
Previous reports have indicated that the apparent position of an external visual target can be affected by nonvisual proprioceptive information about its location provided by touching it with the unseen hand (Lackner, 1976; Lackner \& Zabkar, 1977). This discovery provides a means of evaluating the role of latent vestibular nystagmus in the oculogyral illusion (OGI) (Whiteside, Graybiel, \& Niven, 1965). The OGI is the apparent displacement and motion of a visual target, stationary with respect to an observer, that is seen under conditions of rotary acceleration. Whiteside et al. suggested that perception of body-relative visual displacement in the direction of body acceleration is dependent upon monitoring of the fixation command necessary to override latent nystagmus and thereby maintain a retinally stable image. In the present study, touching a stationary target undergoing illusory displacement with the unseen finger was found to significantly attenuate the illusory displacement associated with the OGI. Moreover, a majority of the subjects experienced a large reduction in the magnitude of their apparent eye movements. These findings support the contention of Whiteside et al. that the OGI results from monitoring the outflow to the extraocular muscles involved in suppressing a covert vestibular nystagmus.
\end{abstract}

Rotational acceleration of a seated individual about his/her longitudinal ( $z$ ) body axis is often used as a way of stimulating the horizontal semicircular canals of the vestibular labyrinth (see Figure 1). If during such stimulation in the dark a visual fixation target is attached to the rotation device, an observer will see it move and displace relative to his/her body in the direction of rotation. Reports of this illusory motion appeared as early as Wells (1792); later descriptions include those of Purkinje (1820), Mach (1886/1959), Barany (1907), and Dusser de Barenne (1934). However, systematic investigation of the phenomenon did not begin until the work of Graybiel and Hupp (1946), who named it the "oculogyral illusion" (OGI).

The OGI has several per- and postrotary characteristics. Initially, during acceleration, the visual target is seen to move and displace relative to the observer in the direction of rotation. Following the acceleration phase and for some period during constant angular velocity, the motion and displacement of the target may appear to reverse direction. With the onset of deceleration, the target appears to move and displace in the direction opposite to that of rotation, and continues to do so for approximately 20-30 sec after cessation of rotation. Under conditions of brisk labyrinthine stimulation, subjects may report additional reversals of perceived target direction during the postrotary phase.

Support for this research was provided by NASA Contract NAS915147. Reprint requests should be addressed to J. N. Evanoff, Krug International, NASA Johnson Space Center, Mail Code KI/SDS NASA Road \#1, Houston, TX 77058.
Most explanations of the OGI have focused on a central misregistration of eye position (e.g., Graybiel \& Hupp, 1946). Nystagmic eye movements are known to occur during rotary acceleration, during some portion of the period of constant velocity, and during deceleration. For the entire acceleration period and for some portion of constant velocity, the nystagmic slow phase is in the direction opposite rotation if a target light is absent. The slow phase of nystagmus reverses direction at the onset of deceleration. When a target light is present and is stationary with respect to the observer, if the observer is unable to fixate it accurately, the slow phase of nystagmus will result in a translation of the stimulus images across the retinas. Assuming that the reflexive, nystagmic eye movements are not centrally registered, this retinal displacement would be interpreted as motion of the target light in the direction of rotation. Under these circumstances, the perceived motion should occur for the duration of nystagmus and a direct relationship should exist between the onset, magnitude, and direction of the OGI and the slow phase of vestibular nystagmus. One must further assume that vision during the fast phase is ignored.

However, this explanation of the OGI encounters several difficulties. The OGI has been shown to commonly outlast postrotational nystagmus by several seconds (Guedry, Collins, \& Sheffey, 1961). The OGI has also been reported during very low acceleration rates in the complete absence of any measurable nystagmus exceeding a few minutes of arc, which suggests that the threshold for the illusion may be somewhat lower than the threshold for overt nystagmus (Cawthorne, Dix, Hallpike, \& Hood, 
1956; Roggeveen \& Hijhoff, 1956). Finally, the illusion has been shown to occur when nystagmus has been inhibited by accurate fixation or in patients suffering temporary paralysis of the extraocular muscles (Battersby, Kahn, Pollack, \& Bender, 1956; Helmholtz, 1925).

Demonstration of an OGI in the absence of significant movements of the eyes led Whiteside, Graybiel, and Niven (1965) to an explanation of the illusion that employed the Helmholtzian notion of " outflow." According to this perspective, "sensations of movement might be associated with a pattern of efferent activity which did not measurably move the eye since there was a fixation point to 'lock-on' to"' (Whiteside et al., 1965, p. 197). Consequently, it is possible to explain the OGI on the basis of a central monitoring of the efferent activity controlling the extraocular muscles antagonistic to the slow phase of vestibular nystagmus; this proposal was recently extended by Post and Leibowitz (1985). If such activity were monitored in determining eye position, then a stationary target should appear to be displaced in the direction opposite the slow phase of the suppressed nystagmus and by an amount proportional to the activity in the extraocular muscles. This is the direction of the displacement component of the OGI. The fact that nystagmus is merely suppressed by fixating a target light can easily be demonstrated by briefly extinguishing the light; when this is done, nystagmus beats reappear.

Two recent studies (Lackner \& Levine, 1978, 1981) showed that the apparent position of an external target light can be affected by nonvisual information about its location, provided by touching it with the unseen hand. The present study was undertaken to determine whether brachial proprioceptive information specifying the position of a visual target could enhance the accuracy of registration of eye position, thereby suppressing the OGI. For example, proprioceptive specification of the target's position might supersede efferent information about the fixation response at oculomotor control centers, thereby resulting in a suppression of the body-relative displacement component of the OGI.

\section{METHOD}

\section{Observers}

Five female and 3 male Brandeis students were paid for their voluntary participation. They had been prescreened to ensure that they had no oculomotor or vestibular deficits that might have affected their performance. None of the subjects had prior experience with the OGI or similar illusions of target motion during labyrinthine stimulation.

\footnotetext{
Apparatus

Labyrinthine stimulation was provided in all conditions by means of a rotating chair that permitted precise alignment of the $z$-axis of the subject's head and torso with the axis of chair rotation (see Figure 1). The rotating chair was equipped with a low-inertia overhead boom assembly with an attached extension in the subject's frontoparallel plane. This boom could be positioned manually by the subject to indicate the apparent spatial location of a light stimulus. The position of the boom was indicated by voltage output from a variable precision potentiometer (linear $\pm .25 \%$ ) affixed at the pivot
}

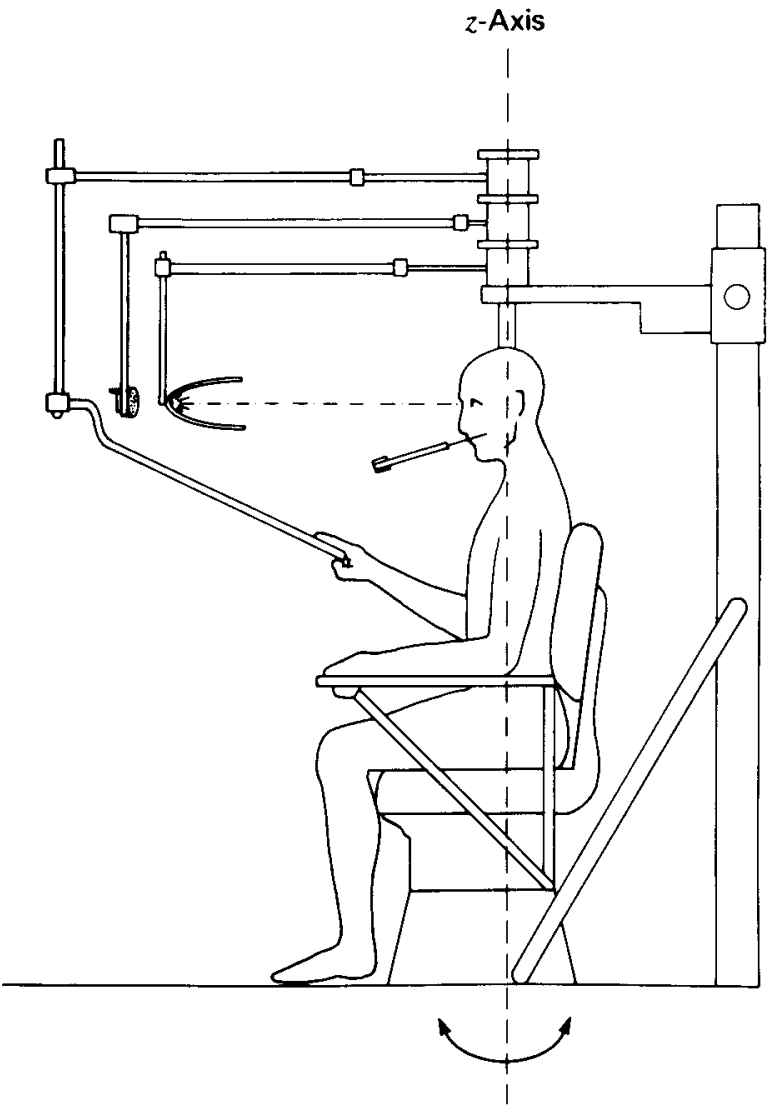

Figure 1. Schematic representation of the rotating chair used to provide labyrinthine stimulation in all experimental conditions.

point. Voltage output was displayed on a separate polygraph channel. Boom position, once calibrated, could be determined within $.1^{\circ}$ of arc.

\section{Procedure}

Four experimental conditions were run in complete darkness with the exception of a single target light, which remained physically stationary with respect to the subject and aligned with an extension of the subjects' objective median plane. The OGI was induced by exposing the subject to trapezoidal angular velocity profiles in a clockwise direction with the $z$-axis of the subject's head and torso aligned with the vertical axis of chair rotation. All subjects participated in all conditions in a repeated measures design. In Condition 1 (standard OGI) the subject's task was to point to the perceived location of the target light with the indicator rod grasped lightly in his/her right hand (see Figure 1). Subjects pointed throughout the trials, from approximately $10 \mathrm{sec}$ preceding the onset of acceleration until they were instructed to discontinue pointing (approximately $30 \mathrm{sec}$ after chair movement ceased). In this manner, a baseline measure of perceived subject-relative target displacement was obtained for each phase of the stimulus profile and a 30-sec aftereffect period. Condition 2 involved the identical tracking task; however, the subject also touched the target light mount (approximately $1 \mathrm{~cm}$ below the light) with the unseen index finger of his/her left hand for the duration of the trial. Immediately preceding each trial, a light intense enough to allow positioning of the finger, but not intense enough to induce an afterimage, was turned on briefly. Condition 3 also incorporated the pointing task; however, in this condition, the index finger of the left hand was positioned approximately $8 \mathrm{~cm}$ immediately behind the target light mount, touching 
a second mount. Finally, in Condition 4 (control), the target light was extinguished and the subject was instructed to hold the pointing rod position constant throughout the entire trial in a random location preset by the experimenter. During this trial the subject's left arm remained on the chair's armrest. This final condition was included to assess effects on the pointing task of involuntary inertial lag of the arm or of vestibulospinal influences on arm control during acceleration and deceleration.

Six clockwise trapezoidal velocity profiles were employed in each of the four experimental çonditions with acceleration rates of 2 , $5,10,15,20$, and 25 degrees per second (dps) squared. These rates were presented to each subject in random order by means of a modified Greco-Latin square such that no condition followed any other condition more than once across all subjects. This assignment of conditions minimized possible effects of adaptation or fatigue. The acceleration and deceleration phases of a profile were identical in each trial and were separated by a constant velocity interval of $45 \mathrm{sec}$. The duration of acceleration was adjusted for each of the six rates to achieve a maximum constant velocity of $100 \mathrm{dps}$. Between trials, a 5-min rest interval provided an opportunity for any after-nystagmus present to subside and for recalibration of eye position. In addition, the rest interval afforded the experimenter an opportunity to query the subjects and collect subjective reports. During all four conditions, each subject's head was held firmly in place, flexed ventrally $25^{\circ}$ and aligned with the body's objective median plane by a dental acrylic bite plate assembly.

The target light (an ultraminiature light-emitting diode, wavelength $530 \mathrm{~nm}$ ), which was present in three of the four conditions, was positioned in alignment with an extension of the subject's median plane at eye level and subtended $20^{\prime}$ of visual angle. Its intensity was adjusted to be photopically effective, but not intense enough to create afterimages.

Conjugate horizontal eye position was monitored continuously throughout each trial by means of conventional dc-coupled electrooculography (EOG). The EOG signals were amplified on the chair by means of a Grass Model P18D preamplifier before being fed through the chair's slipring assembly to a polygraph (Grass Instruments, Model 7D). Electrodes (Harco, HAR-157, silver/silverchloride, pregelled) were positioned on the lateral canthus of each eye with a neutral/ground electrode located on the forehead. The electrodes were allowed to stabilize for $30 \mathrm{~min}$ prior to each condition and subjects were also dark adapted for $10 \mathrm{~min}$ prior to each condition. The gains of the eye polygraph channel and the pointing boom polygraph channel were adjusted during calibration to permit direct comparison of their angular deviations. The chair velocity profile was recorded on a separate polygraph channel.

Each subject's indication of perceived target location was measured in degrees of arc in relation to the target's true position during 1-sec intervals throughout each trapezoidal velocity profile. The arithmetic mean of these displacement values was determined separately for each of the six velocity profiles, and included the 30 -sec period following cessation of rotation.

\section{RESULTS}

The average magnitude of OGI displacement was largest when subjects simply indicated perceived target light position (Condition 1) and smallest when they had the additional proprioceptive information about its true location acquired by touching the target light mount (Condition 2). OGI displacement was of intermediate magnitude when the subject's unseen finger was positioned behind the target light (Condition 3). In the control condition (Condition 4), in which subjects attempted to hold the pointing boom stationary, they were able to do so within $.5^{\circ}$. OGI displacement magnitudes ranged from an average of $10.9^{\circ}$ in Condition 1 to $.1^{\circ}$ in Condition 2. The mean results for all acceleration rates in each of the conditions are graphically represented in Figure 2.

A three-way analysis of variance (ANOVA) performed on these values revealed significant differences among the experimental conditions $[F(3,23)=59.980, p=.00001]$, as well as among the four levels of the trapezoidal profile [acceleration, constant velocity, deceleration, and after-rotation; $F(3,21)=20.816, p=.00001]$. Rate of acceleration was not significant $(p>.45)$. A Tukey test of planned comparisons was performed on all pairwise comparisons within the two significant ANOVA factors to evaluate the nature and extent of the differences. These tests indicated that when brachial proprioceptive cues about target position were present (Condition 2), the displacement component of the OGI was reduced significantly $(p<.01)$. In fact, the apparent target displacements indicated when subjects touched the target mount were not different from displacements obtained in the control condition $(p>.05)$, in which subjects tried to hold the pointing boom stationary. Touching a rod $8 \mathrm{~cm}$ immediately behind the target light (Condition 3) also significantly reduced OGI displacement from baseline OGI $(p<.01)$, but to a lesser extent than in Condition 2 , in which the hand touched the target mount $(p<.01)$; Conditions 2 and 3 were not significantly different from one another $(p>.05)$.

A Tukey test of planned comparisons performed on the four levels of the trapezoidal velocity profile revealed that displacement amplitudes during the constant velocity phase were significantly larger than during the acceleration phase $(p<.01)$, the deceleration phase $(p<.05)$, and the aftereffect period $(p<.01)$. OGI displacements during the aftereffect period were not significantly different from those obtained during acceleration $(p>.05)$ or deceleration $(p>.05)$. Deceleration produced larger OGI displacements than did acceleration $(p<.01)$.

A one-way ANOVA with subjects as a factor revealed a high level of intersubject variability $[F(7,665)=19.274$, $p=.00001]$. An additional ANOVA with gender of the subject as a factor was performed to examine one possible source of this variability. The results indicate that average OGI displacements were significantly larger for female subjects $[F(1,6)=7.693, p<.05]$, with this difference accounting for a large percentage of the intersubject variance.

An analysis of eye movements recorded during all trials in each of the three experimental conditions (Conditions 1,2 , and 3) revealed no discernible ocular deviations in excess of $1.5^{\circ}$ from fixation of the stationary target light, even though the average value of OGI displacements exceeded $10^{\circ}$ in some trials. Subjective reports indicated that the subjects often perceived their eyes to be making large nystagmus-like movements, particularly during acceleration and deceleration in Conditions 1 and 3 . Smaller perceived movements were reported in Condition 2 , although magnitude estimations were difficult to assign. 


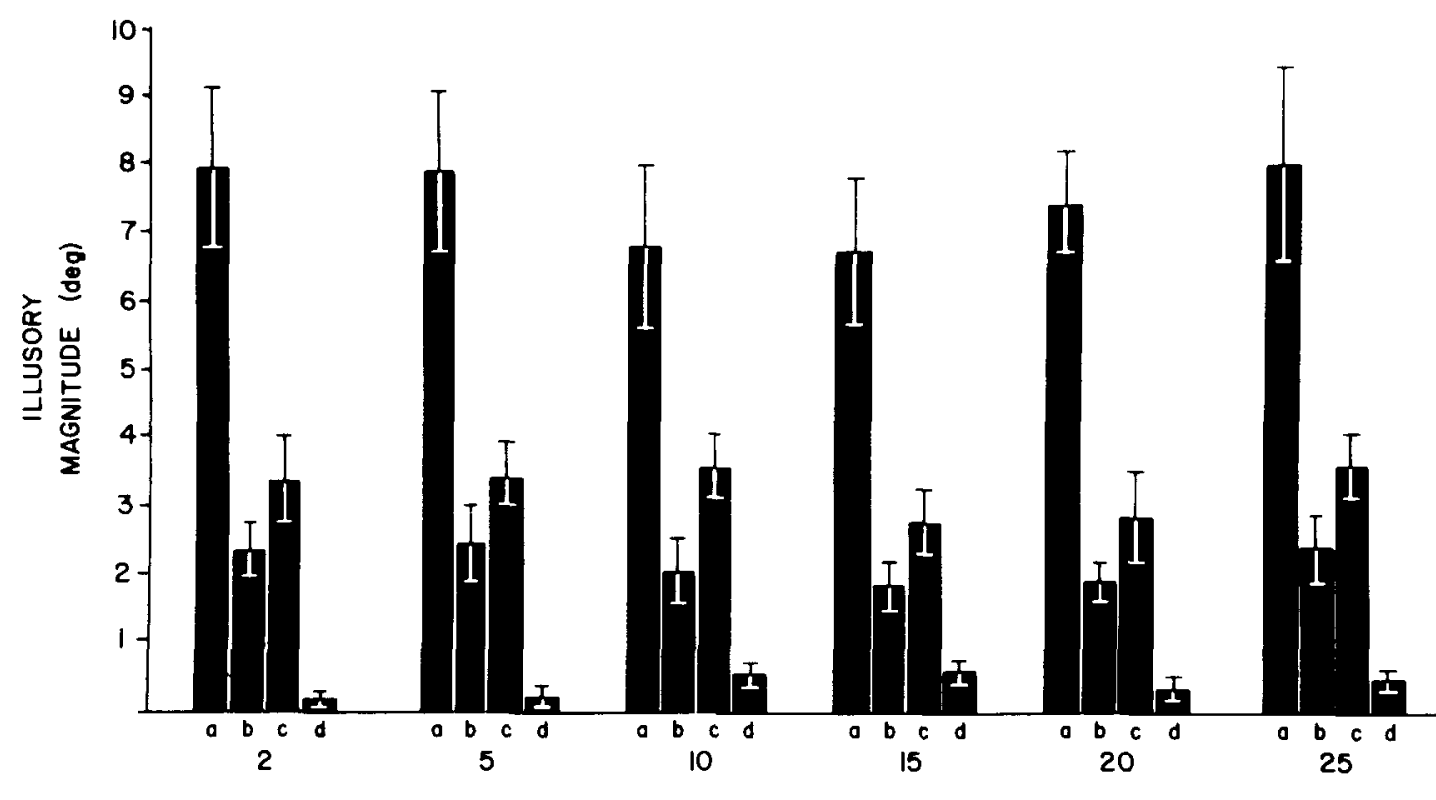

ACCELERATION RATE (deg/sec2)

Figure 2. Mean magnitude indications (in degrees) of perceived OGI displacement for each of six acceleration rates. a, Condition 1-standard OGI: b, Condition 2-perceived OGI while touching target light mount with unseen index finger of left hand; c, Condition 3-perceived OGI while touching a target $8 \mathrm{~cm}$ behind light stimulus mount; d, Condition 4 (control)pointing rod position held stationary. Error bars represent $1 S E M$.

No subject ever reported blurring of the target image or an inability to fixate the light during any trial. In addition, no subject reported perceived motion or displacement of the left arm in Condition 2.

\section{DISCUSSION}

Previous reports have indicated that the perceived location of a distal visual stimulus may be influenced by factors in addition to ocular orientation and the locus of retinal stimulation (Lackner, 1976; Lackner \& Zabkar, 1977). The contributions of these additional sources of sensory information, however, have been largely neglected until very recently. One such factor is proprioceptive information about the relative position of various parts of the body. The present study demonstrated the availability of brachial proprioceptive information and its effectiveness in affecting the perceived visual location of a target light that otherwise would have undergone illusory displacement and movement. In fact, the displacement component of the OGI was reduced approximately $70 \%$ when subjects touched the visual target mount.

The apparent target displacement associated with the OGI appears to involve a central misinterpretation of eye position. Under all experimental conditions, the visual stimulus was quite accurately foveated, due to the voluntary fixation response that suppressed vestibular nystagmus. The central interpretation of target location, as reflected in OGI displacement, appears to involve and parallel this fixation command. Evanoff and Lackner
(1987) recently showed, for example, that variations in fixation-signal strength necessary to maintain stable target fixation resulted in systematic and predictable differences in OGI magnitude. That this happened in the present study is apparent from spontaneous reports by several subjects that in Conditions 1 and 3 they experienced large deviations of their eyes in the direction of apparent target displacement during and after rotation. The eye movement recordings indicated that the subjects were actually maintaining accurate fixation of the stationary target during these conditions. When the subjects were allowed to touch the target light mount, the perceived displacement of the target was significantly reduced from the normally occurring OGI. Moreover, a majority of the subjects experienced a large reduction in the magnitude of their apparent eye movements; and 2 reported that their eyes seemed to remain stationary. These reports by subjects are consistent with earlier findings of Evanoff and Lackner (1985) that brachial proprioceptive information was of sufficient fidelity to contribute to a fixation response adequate to suppress both the slow and fast phases of vestibular nystagmus. Clearly, a sensory input adequate to override vestibular nystagmus and the displacement component of the OGI need not originate in the visual modality. These findings support the original contention of Whiteside et al. (1965) that the OGI results from monitoring the outflow to the extraocular muscles involved in suppressing a covert vestibular nystagmus.

When the subject's hand was positioned directly behind the target light (Condition 3), the magnitude of the OGI 
was larger than when the target mount was touched (Condition 2). This increase as a function of the distance of the unseen hand from the target suggests that the proprioceptive influence on visual direction is dependent upon target and hand coincidence. This dependence is in accord with similar observations by Lackner and Shenker (1985), who found that the influence of apparent arm position on visual and auditory direction was greatest when there was coincidence of hand and target. How the proprioceptive influence decreases as a function of arm distance from the target, especially when the hand is positioned laterally in relation to the target, remains to be quantified.

The appearance of a marked gender difference in the magnitude of the OGI in Condition 1 (standard) was unexpected. However, most earlier research on the OGI used only male subjects, and this difference would not have been noticed. In the current study, females reported OGI magnitudes nearly twice as large as those reported by males (suppression was nearly complete for both sexes when they touched the target light mount). Potential explanations for this difference are highly speculative and relate to gender differences in oculomotor motility, internal representations of the body's center of gravity, inertial characteristics of the body, or vestibular ocular reflex (VOR) gain (see Wall, Black, \& Hunt, 1984).

\section{REFERENCES}

Barany, R. (1907). Physiologie und Pathologie des Bogengang Apparates beim Menschen. Vienna: Franz Deuticke.

Battersby, W. S., Kahn, R. L., Pollack, M. R., Bender, M. B. (1956). Effects of visual, vestibular and somatosensori-motor deficit on autokinetic perception. Journal of Experimental Psychology, 52, 398-410.

CaWthorne, T., Dix, M. R., Hallpike, G. S., Hood, J. D. (1956). The investigation of vestibular function. British Medical Bulletin, 12, 131-133.

Dusser De BAREnNe, J. G. (1934). The labyrinthine and postural mechanisms. A handbook of general experimental psychology. Worcester, MA: Clark University Press.

Evanoff, J. N., \& Lackner, J. R. (1985). Proprioceptive influences on vestibular nystagmus and the displacement component of the oculogyral illusion. Society for Neuroscience Abstracts, 11, No. 207.17.
EVANOFF, J. N., \& LACKNER, J. R. (1987). Influence of maintained ocular deviation on the spatial displacement component of the oculogyral illusion. Perception \& Psychophysics, 42, 25-28.

Graybiel, A., \&UPP, D. (1946). The oculo-gyral illusion; a form of apparent motion which may be observed following stimulation of the semi-circular canals. Joumal of Aviation Medicine, 17, 1-12.

Guedry, F. E., Coluns, W. E., \& Sheffey, P. L. (1961). Perceptual and oculomotor reactions to interacting visual and vestibular stimulation. (Report No. 463, USAMRL Proj. 6X95-25-001-15). Fort Knox, KY: U.S. Army Medical Research Laboratory.

HeLMHOLTz, H. L. F. voN. (1925). Helmholtz's treatise on physiological optics: Vol. 3. The perception of vision (J. P. C. Southall, Ed. and Trans.). Rochester, NY: Optical Society of America.

LACKNER, J. R. (1976). Influence of abnormal postural and sensory conditions on human sensory motor performance. Environmental Biology \& Medicine, 2, 139-177.

LACKNER, J. R., \& LEVINE, M. S. (1978). Visual direction depends on the operation of spatial constancy mechanisms. Neuroscience Letters, 7, 207-212.

LACKNER, J. R., Levine, M. S. (1981). The guidance of saccadic eye movements to perceptually mislocalized visual and non-visual targets. Aviation, Space \& Environmental Medicine, 52, 461-465.

LACKNer, J. R., Shenker, B. (1985). Proprioceptive influences on auditory and visual spatial localization. Journal of Neuroscience, $\mathbf{3}$, 579-583.

LACKNer, J. R., \& Zabkar, J. J. (1977). Proprioceptive information about target location suppresses autokinesis. Vision Research, 17, 1225-1229.

MACH, E. (1959). The analysis of sensations (J. Fisher, Trans.). New York: Dover. (Original work published 1886)

Post, R. B., LeIBowitz, H. W. (1985). A revised analysis of the role of efference in motion perception. Perception, 14, 631-643.

Purkinje, J. E. (1820). Beitrage zur mehreren Kenntnis des Schiuindels aus heautogenostischen Daten. Med. Jb. (Austria), 6, 79-125.

RogGeveEN, L. I., \&uHofF, P. (1956). The normal and pathological threshold of the perception of angular accelerations for the optogyral illusion and the turning sensations. Acta Oto-Laryngologica, 46, 533-541.

Wall, C., III, Black, F. O., Hunt, A. E. (1984). Effects of age, sex and stimulus parameters upon vestibulo-ocular responses to sinusoidal rotation. Acta Oto-Laryngologica, 98, 270-278.

WeLLS, W. C. (1792). An essay upon single vision with two eyes together with experiments and observations on several other subjects in optics. London: Cadell.

Whiteside, T. C. D., Graybiel, A., Niven, J. I. (1965). Visual illusions of movements. Brain, 88, 193-219.

(Manuscript received December 22, 1986; revision accepted for publication June 23,1987 .) 